\title{
Entropy in the interior of a black hole and thermodynamics
}

\author{
Baocheng Zhang* \\ School of Mathematics and Physics, \\ China University of Geosciences, Wuhan 430074, China
}

\begin{abstract}
Based on a recent proposal for the volume inside a black hole, we calculate the entropy associated with this volume and show that such entropy is proportional to the surface area of the black hole. Together with the consideration of black hole radiation, we find that the thermodynamics associated with the entropy is highly possible to be caused by the vacuum polarization near the horizon.
\end{abstract}

*Electronic address: zhangbc.zhang@yahoo.com 


\section{INTRODUCTION}

How big is a black hole? This is not an easy question, since the definition of the volume of the space inside a black hole depends on how the spacetime is sliced into space and time, unlike the surface area of the black hole that is the same for all observers. Intuitively, a nice description for the volume should be slicing invariant, which was made firstly by Parikh [1] and also discussed by others [2 6]. Recently, a different method was suggested by Christodoulou and Rovelli [7] based on a simple observation that the interior of Schwarzschild black holes is not static, which leaded to another sensible description for the volume as the largest volume bounded by the event horizon of a black hole. For a Schwarzschild black hole, they showed that at late time the volume took such an expression,

$$
V_{C R} \sim 3 \sqrt{3} \pi M^{2} v
$$

where $M$ is the mass of the black hole and $v$ is the advanced time. In the paper, we will call the volume as CR volume for brevity. Instantly, this result was extended to other backgrounds of spacetime [8-10].

Relative to the specific forms for the volume inside a black hole, ones concern more about its significance, that is, why do we want to investigate the volume? This was regarded to be relevant to the black hole information loss paradox in past studies. So it is unavoidable to involve the black hole radiation [11] and the corresponding thermodynamics [12, 13] in such studies, but up to now they only were involved with the qualitative discussions. In this paper, we will try to make some quantitative calculations to estimate the entropy associated with the CR volume.

As well-known, a black hole can emit the thermal radiation that makes the black hole have a lifetime $\sim M^{3}$ and thus the $\mathrm{CR}$ volume inside the black hole has an extraordinary form that is proportional to $M^{5}$. A natural question arises: for such a large volume, how many field modes can be included in it. Furthermore, whether these modes are relevant to the interpretation of Bekenstein-Hawking entropy statistically. When the radiation happens, the

background geometry will be altered according to the semiclassical Einstein equation, which, in equilibrium, can be described with the first law of black hole thermodynamics. Thus one might ask: whether the maximal volume is also included in the background geometry. If the volume changes, how is the first law changed? In this paper, we will work on these problems. 
The structure of the paper is as follows. Firstly, we will revisit the definition of the volume inside a black hole by Christodoulou and Rovelli, and present explicitly the choice of the maximal hypersurfaces [14] by maximal slicing [15, 16] in the second section. Then, we calculate the entropy in the volume using the standard statistical method in the third section. In the forth section, we discuss the first law of black hole thermodynamics, in particular for the newly obtained entropy, which is related to the vacuum polarization [17] near the horizon of the black hole. Finally, we will give a conclusion in the five section.

\section{BLACK HOLE VOLUME AND MAXIMAL SLICING}

Start with the geometry of a collapsed object as in Ref. [7], which can be described with the Eddington-Finkelstein coordinates,

$$
d s^{2}=-f(r) d v^{2}+2 d v d r+r^{2} d \theta^{2}+r^{2} \sin ^{2} \theta d \phi^{2}
$$

where $f(r)=1-\frac{2 M}{r}$ and the advanced time $v=t+\int \frac{d r}{f(r)}=t+r+2 M \ln |r-2 M|$. In particular, we have taken the units $G=c=\hbar=k_{B}=1$. The advantage of the coordinates over the static one is that there is no coordinate singularity at the event horizon. Thus it can be analytically continued to all $r>0$, which is required for the description of the geometry of the collapsed matter.

In order to calculate the volume, a proper hypersurface has to be chosen. With an transformation $v \rightarrow v(T, \lambda), r \rightarrow r(T, \lambda)$, the coordinates (2) becomes $d s^{2}=$ $\left(-f\left(\frac{\partial v}{\partial T}\right)^{2}+2 \frac{\partial v}{\partial T} \frac{\partial r}{\partial T}\right) d T^{2}+\left(-f\left(\frac{\partial v}{\partial \lambda}\right)^{2}+2 \frac{\partial v}{\partial \lambda} \frac{\partial r}{\partial \lambda}\right) d \lambda^{2}+r^{2} d \theta^{2}+r^{2} \sin ^{2} \theta d \phi^{2}$ where we have assumed the cross term vanishes by taking the transformation properly. In particular, if the condition $-f\left(\frac{\partial v}{\partial T}\right)^{2}+2 \frac{\partial v}{\partial T} \frac{\partial r}{\partial T}=-1$ (i.e. this can be realized by taking $d v=\frac{-1}{\sqrt{-f}} d T+d \lambda, d r=$ $\sqrt{-f} d T$ which also removes the cross term simultaneously) is enforced, one will find that the hypersurface $\Sigma: T=$ constant, is that chosen in Ref. [7] where the spherically symmetric hypersurface is taken as the direct product of a 2-sphere and an arbitrary curve parameterized by $\lambda$ in $v-r$ plane. In particular, it is noted that the hypersurface $T=$ constant is able to be gotten by $r=$ constant according to the transformation $d r=\sqrt{-f} d T$.

The hypersurface $\Sigma$, as in Ref. [7], is coordinatized by $\lambda, \theta, \phi$, and the line element of the induced metric on it can be expressed as

$$
d s_{\Sigma}^{2}=\left(-f(r) \dot{v}^{2}+2 \dot{v} \dot{r}\right) d \lambda^{2}+r^{2} d \theta^{2}+r^{2} \sin ^{2} \theta d \phi^{2}
$$


where the dot represents the partial derivative with regard to the parameter $\lambda$, and $-f(r) \dot{v}^{2}+$ $2 \dot{v} \dot{r}>0$ for the spacelike hypersurface. The maximal volume can be obtained by the integral,

$$
V_{\Sigma}=4 \pi \int d \lambda \sqrt{r^{4}\left(-f(r) \dot{v}^{2}+2 \dot{v} \dot{r}\right)} .
$$

with a proper choice of the curves. An investigation for geodesics in an auxiliary manifold gave the maximization condition by choosing the curves [7],

$$
r=\frac{3}{2} M,
$$

which, together with Eq. (44), gives the CR volume expressed in Eq. (1).

On the other hand, the maximization can also be calculated in mathematical relativity [18] where a method called as maximal slicing can lead to hypersurfaces of maximal volume which have vanishing mean extrinsic curvature, $K=0$, where $K$ is the trace of the extrinsic curvature of the hypersurface. Here we show the vanishing $K$ is equivalent to the condition (5). According to Ref. [16], we use the coordinates (2) and take the spacelike hypersurfaces by $r=$ constant, since the time and space are regarded as being interchanged across the horizon of a Schwarzschild black hole. Take $n$ as the future pointing timelike unit normal to the hypersurfaces $\Sigma_{r}$,

$$
n=\sqrt{-f}\left(\frac{\partial}{\partial r}+\frac{1}{f} \frac{\partial}{\partial v}\right) .
$$

It is easy to confirm that $g_{\mu \nu} n^{\mu} n^{v}=-1$ for the coordinates (2). It is noted that there is a simple relation between the divergence of the vector $n$ and the trace of the extrinsic

curvature tensor $K_{\mu \nu}, K=-\nabla \cdot n$ where $\nabla$ is the covariant derivative with respect to the spacetime metric. With the given metric (2), we have

$$
K=\frac{1}{2 \sqrt{-f}}\left(\frac{\partial f}{\partial r}+4 f \frac{1}{r}\right)=0,
$$

which gives the equation $r=\frac{3}{2} M$, consistent with the condition (5).

\section{ENTROPY IN THE VOLUME}

Since the CR volume is obviously different from the normal volume that might still exist inside the black hole, it is significant to investigate how many modes of quantum fields can be included in CR volume. In the paper, we involves only the scalar field. According to the standard quantum statistical method [19], the number of quantum states in some volume 
has to be counted for one certain phase-space which can be labeled here by $\lambda, \theta, \phi, p_{\lambda}, p_{\theta}, p_{\phi}$. From the uncertainty relation of quantum mechanics, $\Delta x_{i} \Delta p_{i} \sim 2 \pi$, one quantum state corresponds to a "cell" of volume $(2 \pi)^{3}$ in the phase-space. Therefore, the number of quantum states is given by

$$
\frac{d \lambda d \theta d \phi d p_{\lambda} d p_{\theta} d p_{\phi}}{(2 \pi \hbar)^{3}} .
$$

In order to calculate the integral, we consider a massless scalar field $\Phi$ in the spacetime with the coordinates,

$$
d s^{2}=-d T^{2}+\left(-f(r) \dot{v}^{2}+2 \dot{v} \dot{r}\right) d \lambda^{2}+r^{2} d \theta^{2}+r^{2} \sin ^{2} \theta d \phi^{2}
$$

It is noted that this metric is equivalent to such an form: $d s^{2}=-d T^{2}+H(T) d \lambda^{2}+r(T)^{2} d \theta^{2}+$ $r(T)^{2} \sin ^{2} \theta d \phi^{2}$, which means that it is not static for the defined time $T$ in the interior of the black hole. For this reason, sometimes the interior of the black hole is interpreted as a cosmological model (see also Ref. [20]), and it evolves towards the singularity of the black hole. It is not entirely clear what should be done for the statistics in a dynamic background, but fortunately, our calculation is made at $v>>M$ and $r=\frac{3}{2} M$. For the maximal slicing, the slices accumulate on a limiting hypersurface $r=\frac{3}{2} M$ when $t$ is large enough (that is guaranteed by $v>>M$ ) [15]. That is to say, near the maximal hypersurface, the proper time between two neighbouring hypersurfaces tends to zero as $t$ increases, so nearly no evolution happened there. Thus our statistical calculation is not affected by the non-static character of the metric, since it is calculated on approximately $T=$ constant which is the hypersurface that leads to the CR volume. Therefore, in what follows we will use the common method in the curved spacetime to discuss the motion of scalar field in the interior of the black hole.

Using the WKB approximation, the field $\Phi$ can be written as $\Phi=$ $\exp [-i E T] \exp [i I(\lambda, \theta, \phi)]$, and then substituting it into the Klein-Gordon equation in curved spacetime, $\frac{1}{\sqrt{-g}} \partial_{\mu}\left(\sqrt{-g} g^{\mu \nu} \partial_{\nu} \Phi\right)=0$, we obtain

$$
E^{2}-\frac{1}{-f(r) \dot{v}^{2}+2 \dot{v} \dot{r}} p_{\lambda}^{2}-\frac{1}{r^{2}} p_{\theta}^{2}-\frac{1}{r^{2} \sin ^{2} \theta} p_{\phi}^{2}=0
$$

where $p_{\lambda}=\frac{\partial I}{\partial \lambda}, p_{\theta}=\frac{\partial I}{\partial \theta}, p_{\phi}=\frac{\partial I}{\partial \phi}$. Thus, following the Eq. (6) 
with the energy less than $E$ is obtained as

$$
\begin{aligned}
g(E) & =\frac{1}{(2 \pi)^{3}} \int d \lambda d \theta d \phi d p_{\lambda} d p_{\theta} d p_{\phi} \\
& =\frac{1}{(2 \pi)^{3}} \int \sqrt{-f(r) \dot{v}^{2}+2 \dot{v} \dot{r}} d \lambda d \theta d \phi \int \sqrt{E^{2}-\frac{1}{r^{2}} p_{\theta}^{2}-\frac{1}{r^{2} \sin ^{2} \theta} p_{\phi}^{2}} d p_{\theta} d p_{\phi} \\
& =\frac{1}{(2 \pi)^{3}} \int \sqrt{-f(r) \dot{v}^{2}+2 \dot{v} \dot{r}} d \lambda d \theta d \phi\left(\frac{2 \pi}{3} E^{3} r^{2} \sin \theta\right) \\
& =\frac{E^{3}}{12 \pi^{2}}\left[4 \pi \int d \lambda \sqrt{r^{4}\left(-f(r) \dot{v}^{2}+2 \dot{v} \dot{r}\right)}\right] \\
& =\frac{E^{3}}{12 \pi^{2}} V_{C R},
\end{aligned}
$$

where the relation $p_{\lambda}=\sqrt{-f(r) \dot{v}^{2}+2 \dot{v} \dot{r}} \sqrt{E^{2}-\frac{1}{r^{2}} p_{\theta}^{2}-\frac{1}{r^{2} \sin ^{2} \theta} p_{\phi}^{2}}$ is used in the second line, the integral formula $\iint \sqrt{1-\frac{x^{2}}{a^{2}}-\frac{y^{2}}{b^{2}}} d x d y=\frac{2 \pi}{3} a b$ is used in the third line, and in the final line we have used the condition (5). As expected, the number of quantum states is proportional to the $\mathrm{CR}$ volume, but this is still different from a normal situation, because the $\mathrm{CR}$ volume is the result of the curved spacetime.

Temporarily ignoring the exotic feature of CR volume, we can continue to calculate the free energy at some inverse temperature $\beta$,

$$
\begin{aligned}
F(\beta) & =\frac{1}{\beta} \int d g(E) \ln \left(1-e^{-\beta E}\right) \\
& =-\int \frac{g(E) d E}{e^{\beta E}-1} \\
& =-\frac{V_{C R}}{12 \pi^{2}} \int \frac{E^{3} d E}{e^{\beta E}-1} \\
& =-\frac{\pi^{2} V_{C R}}{180 \beta^{4}} .
\end{aligned}
$$

Furthermore, the entropy is obtained as

$$
S_{C R}=\beta^{2} \frac{\partial F}{\partial \beta}=\frac{\pi^{2} V_{C R}}{45 \beta^{3}}
$$

which looks like the entropy in the normal volume.

Now we turn to the CR volume again, but under the consideration with Hawking radiation. Since Hawking radiation is thermal, the loss mass rate of a Schwarzschild black hole can be given by the Stefan-Boltzmann law,

$$
\frac{d M}{d v}=-\frac{1}{\gamma M^{2}}, \gamma>0
$$


where $\gamma$ is a constant whose value does not influence the discussion in the paper. In Ref. [10], it is discussed that the large volume is remained until the final stage of black hole evaporation. Here we are concerned about the time that the radiation can last. Thus, for a black hole with the mass $M$, we have

$$
v \sim \gamma M^{3}
$$

which also satisfies the requirement in Ref. [7], $v>>M$. Then using the Eqs. (11) and (11), and considering the temperature for a Schwarzschild black hole, $\beta=T^{-1}=8 \pi M$, one find the entropy

$$
S_{C R} \sim \frac{(3 \sqrt{3} \gamma) M^{2}}{\left(45 \times 8^{3}\right)}=\frac{(3 \sqrt{3} \gamma)}{\left(90 \times 8^{4}\right) \pi} A,
$$

where $A=16 \pi M^{2}$ is the surface area of the Schwarzschild black hole. This is a surprising and intriguing result that the entropy from quantum theory in the $\mathrm{CR}$ volume is proportional to the surface area of the black hole horizon that bounds the volume. Note that the result is dependent on the validity of the relation (12), which was shown [21] to hold so long as the mass of a Schwarzschild black hole is greater than the Planck mass. Moreover, the exact description of final stage of black hole evaporation has not existed, but some specific considerations such as General Uncertainty Relation (i.e. see the review [22]) implied that at the final stage, the loss mass rate becomes $\frac{d M}{d v} \sim M$ [23], which is inconsistent with the requirement for the calculation of the $\mathrm{CR}$ volume, beyond our problem obviously. On the other hand, although the entropy $S_{C R}$ is proportional to the surface area, a rough estimation finds that the parameter before the area is much smaller than $\frac{1}{4}$. Then a natural problem: what is the entropy $S_{C R}$ ? Whether it will be included in the first law of black hole thermodynamics. In what follows, we will discuss it.

\section{FIRST LAW AND VACUUM POLARIZATION}

Since the derivation of $\mathrm{CR}$ volume is in the case of $v>>M$, which means the black hole has formed by the collapse of the matter and is static for the external observers. Thus, due to the Hawking radiation, the first law of black hole thermodynamics reads as

$$
d M=T d S_{H}
$$

where the entropy $S_{H}=\frac{1}{4} A$. This relation was obtained [11, 12] by the analogy of black hole physics to thermodynamics which will reach an equilibrium state after the relaxation 
processes are completed. The change of the entropy $S_{H}$ is equivalent to the change of the surface area, so the entropy $S_{C R}$ in the CR volume will be changed. Although our earlier analysis shows that the entropy $S_{C R}$ is proportional to the surface area, its original form in Eq. (11) is closely related to the CR volume. So in equilibrium, the thermal process requires a term relevant to the change of $\mathrm{CR}$ volume. According to the general thermodynamics, such term should be written as $P d V_{C R}$ where $P$ is the pressure. Thus, we expect that the first law can be written as

$$
d M=T d S-P d V_{C R},
$$

where $S=S_{H}+S_{C R}$. In order to remain the validity of the first law, a relation is required, $T d S_{C R} \sim P d V_{C R}$. Now, the vital problem is that from where and how much the pressure is, after the collapsed matter had been concentrated into the singularity (it is also noted that some studies pointed out that the nonlocal effect will prevent the black hole from collapsing into a physical singularity, i.e. see Ref. [24]).

In the original calculation of Hawking [11], the concept of particles are used in the asymptotically flat region far from the black hole where they can be unambiguously defined. While the particle flux carries away the positive energy, an accompanying flux of negative energy goes into the hole across the horizon, which can only be understood by the zero point fluctuations of local energy density in the quantum theory. This phenomena, called also as vacuum polarization, will play an important role in the neighborhood of the black hole.

The vacuum polarization is usually considered in the semi-classical Einstein gravity, in which the fluctuations of gravitational field are small and so is the expectation value of the energy-momentum tensor of the relevant quantized fields in the chosen vacuum state. By solving the modified Einstein equation, $G_{\mu \nu}=8 \pi\left\langle T_{\mu \nu}\right\rangle$, the quantum pressure at the horizon caused by the vacuum polarization is given by [25 28],

$$
P=\frac{1}{\left(90 \times 8^{4}\right) \pi^{2}} \frac{1}{M^{4}} .
$$

It is noted that the CR volume is calculated for the hypersurface $r=\frac{3}{2} M$, while $P$ is at the horizon. But it is not difficult to clarify this, since the $\mathrm{CR}$ volume is just the volume for the black hole, and the boundary for the volume is still the horizon. This point was also seen in Ref. [7], by the parameter $\lambda$ that take the range covering the region from $r=0$ to $r=2 M$. In particular, some recent interesting studies considered the pressure as stemming 
from the cosmological constant, i.e. see Refs. [29-31], but they are different from ours since the cosmological constant is not involved here.

The term $P d V_{C R}$ is calculated as

$$
P d V_{C R}=\frac{5(3 \sqrt{3} \gamma)}{\left(90 \times 8^{4}\right) \pi} d M
$$

which is on the level of $10^{-5}$ for the parameter before $d M$, and is very small, as expected. The term $T d S_{C R}$ is also easy to be estimated as

$$
T d S_{C R}=\frac{4(3 \sqrt{3} \gamma)}{\left(90 \times 8^{4}\right) \pi} d M \sim 10^{-5} d M .
$$

It is seen that the term $T d S_{C R}$ is not equal exactly to the term $P d V_{C R}$, but fundamentally they can be canceled on the level of $10^{-5}$. The reason is that the values of the pressure $P$ and the temperature $T$ is not so exact in the present consideration. Actually, a direct evidence that the thermodynamics in the $\mathrm{CR}$ volume is caused by vacuum polarization is that the term $P d V_{C R}$ is not dependent on the black hole parameter $M$, as obtained for the term $T d S_{C R}$.

Finally, it is noted that if taking such a term $V_{C R} d P$, as made in Refs. [29 31], it can balance exactly the change of thermodynamics caused by $T d S_{C R}$, but no evidence shows that why the pressure here should be changed, so the exact cancelation might be occasional. In fact, vacuum polarization causes the quantum correction to the usual black hole thermodynamics, so the corresponding thermodynamic variables would be corrected, which is the reason why the terms $P d V_{C R}$ and $T d S_{C R}$ cancel each other out approximately.

\section{CONCLUSION}

In the paper, we have investigated the relation between the derivation of the volume of the space inside a Schwarzschild black hole defined by Christodoulou and Rovelli and the maximal slicing, and found explicitly that the CR volume was just obtained for the hypersurface whose mean extrinsic curvature is zero. We have also calculated the entropy in the $\mathrm{CR}$ volume through counting the number of quantum states in the volume with a standard statistical method. Differently from the normal situation, the entropy associated with the CR volume is proportional to the surface area of the black hole, but the parameter is

much smaller than that required for the Bekenstein-Hawking entropy. The small parameter 
has also been interpreted in the paper, from the perspective of black hole thermodynamics, for which a suggestive result is given that the thermodynamics associated with the entropy in the $\mathrm{CR}$ volume is caused by the vacuum polarization near the horizon, since the matter has collapsed into the singularity when we investigate this phenomena. Thus, our result verifies further the relation between black hole physics and quantum theory again.

\section{ACKNOWLEDGE}

The author would like to thank Prof. C. Rovelli for reading this paper and his positive comments. This work is supported by Grant No. 11374330 of the National Natural Science

Foundation of China and by Open Research Fund Program of the State Key Laboratory of Low-Dimensional Quantum Physics and by the Fundamental Research Funds for the Central Universities, China University of Geosciences (Wuhan)(CUG150630).

[1] M. K. Parikh, Phys. Rev. D 73, 124021 (2006).

[2] D. Grumiller, arXiv: 0509077 [gr-qc].

[3] B. S. DiNunno and R. A. Matzner, Gen. Relativ. Gravi. 42, 63 (2009).

[4] W. Ballik and K. Lake, arXiv: 1005.1116 [gr-qc].

[5] M. Cvetič, G. W. Gibbons, D. Kubiznák, and C. N. Pope, Phys. Rev. D 84, 024037 (2011).

[6] W. Ballik and K. Lake, Phys. Rev. D 88, 104038 (2013).

[7] M. Christodoulou and C. Rovelli, Phys. Rev. D 91, 064046 (2015).

[8] I. Bengtsson, E. Jakobsson, arXiv:1502.01907 [gr-qc].

[9] Y. C. Ong, JCAP 04, 003 (2015).

[10] Y. C. Ong, arXiv:1503.08245 [gr-qc].

[11] S. W. Hawking, Nature 248, 30 (1974); S. W. Hawking, Commun. Math. Phys. 43, 199 (1975).

[12] J. D. Bekenstein, Phys. Rev. D 7, 2333 (1973).

[13] J. M. Bardeen, B. Carter, and S. W. Hawking, Commun. Math. Phys. 31, 161 (1973).

[14] J. E. Marsden and F. J. Tipler, Phys. Rep. 66109 (1980).

[15] F. Estabrook, H. Wahlquist, S. Christensen, B. DeWitt, L. Smarr, and E. Tsiang, Phys. Rev. D 7, 2814 (1973). 
[16] I. Cordero-Carrión, J. M. Ibáñez, and J. A. Morales-Lladosa, J. Math. Phys. 52, 112501 (2011).

[17] V. P. Frolov and I. D. Novikov, Black Hole Physics: Basic Concepts and New Developments (Kluwer Academic Publishers, Dordrecht, Netherlands, 1998).

[18] E. Gourgoulhon, arXiv: qr-qc/0703035

[19] B. Cowan, Topics in Statistical Mechanics (Royal Holloway, Imperial College Press, London, UK, 2005).

[20] S. M. Carroll, M. C. Johnson, and L. Randall, J. High Energy Phys. 11 (2009) 094.

[21] S. Massar, Phys. Rev. D 52, 5857 (1995).

[22] S. Hossenfelder, Living Rev. Relativity 16, 2 (2013).

[23] L. Xiang, Phys. Lett. B 540, 9 (2002).

[24] A. Saini and D. Stojkovic, Phys. Rev. D 89044003 (2014).

[25] W. G. Unruh, Phys. Rev. D 14, 870 (1976).

[26] P. Candelas, Phys. Rev. D 21, 2185 (1980).

[27] D. N. Page, Phys. Rev. D 25, 1499 (1982).

[28] T. Elster, Phys. Lett. A 94, 205 (1983).

[29] D. Kastora, S. Rayb, and J. Traschena, Classical Quantum Gravity 26, 195011 (2009).

[30] B. P. Dolan, Classical Quantum Gravity 28, 235017 (2011).

[31] D. Kubiznak and R. B. Mann, arXiv: 1404.2126. 